\title{
Electron Channelling Contrast Imaging (ECCI): An Amazing Tool for Observations of Crystal Lattice Defects in Bulk Samples
}

Stefan Zaefferer ${ }^{1}$

\section{${ }^{1}$ Max Planck Institute for Iron Research, Dusseldorf, Germany}

Electron channelling is a contrast mechanism in scanning electron microscopy (SEM) which allows, using backscattered electrons, direct observation of crystal lattice defects like dislocations, stacking faults, strain fields and others in a volume close to the surface of a bulk sample. Although the technique is long-time known it is only with the advent of suitable microscopes that it can be fully exploited. In particular it is important that the instrument, like a scanning transmission electron microscope (STEM), delivers a beam with very small beam convergence, high brightness and small spot size. Furthermore, the backscatter detector must be large and quick. All these contradicting requirements are fulfilled with certain modern field emission gun SEMs.

ECC arises due to the modulation of the backscattering yield by the crystal lattice. In fact, electrons travel in a crystal lattice in form of a standing wave. For situations where the primary electrons enter the lattice close to the Bragg angle of some set of lattice planes, the maxima of this standing electron wave field are situated between the atoms which leads to very little interaction of the primary electrons with the lattice and consequently a low backscattering yield. This case is called electron channeling. The opposite case arises when the beam electrons travel close to parallel to the lattice planes. In this case the maxima of the wave field or focussed onto the atoms. which results in intense interaction and a high backscattering yield. The presence of lattice defects in a crystal illuminated in channelling mode leads to an increased backscattering yield and to the occurrence of electron channelling contrast at these defects.

The fact that the contrast arises only from that area where the electron beam pursues a welldefined direction with the lattice results in a very good spatial resolution of the technique, being in the order of $8 \mathrm{~nm}$ lateral and about 50 to $100 \mathrm{~nm}$ into the depth of the material for a well-collimated beam of $30 \mathrm{keV}$ electrons.

Using ECCI it is possible to observe lattice defects, in a number of different materials, including all kinds of metals, ceramics and even some minerals. Since the samples are observed in bulk form and not as thin foils, the defects are potentially seen in a more natural arrangement because no stress release occurs during sample preparation. Furthermore, the bulk nature of the samples allows performing straining or heating experiments more easily as with TEM samples.

We will present a short overview on the theory of ECCI [1], followed by some examples indicating the practice of the technique [1] like slip bands in high entropy alloys (Fig. 1). We will show some further advanced applications like Burgers vector determination in conjunction with hydrogen embrittlement of superalloys and nanoindentation in austenitic steels (Fig. 2). Finally the contrast of ECCI will be discussed in conjunction with calculated dislocation images. 


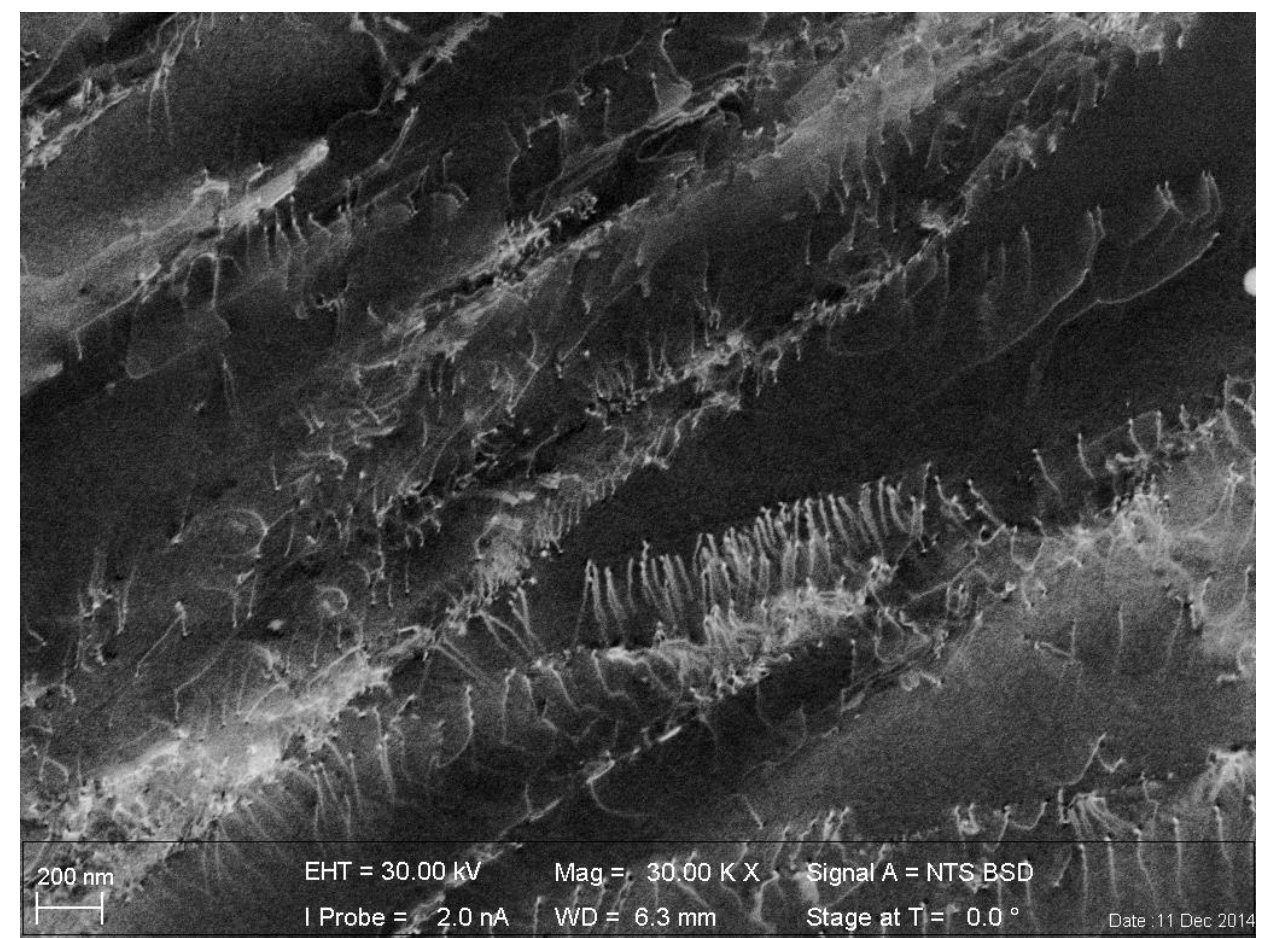

Figure 1: Slip bands consisting of individual dislocations observed in a high entropy alloy. Sample is a courtesy of N. Wanderka, Berlin

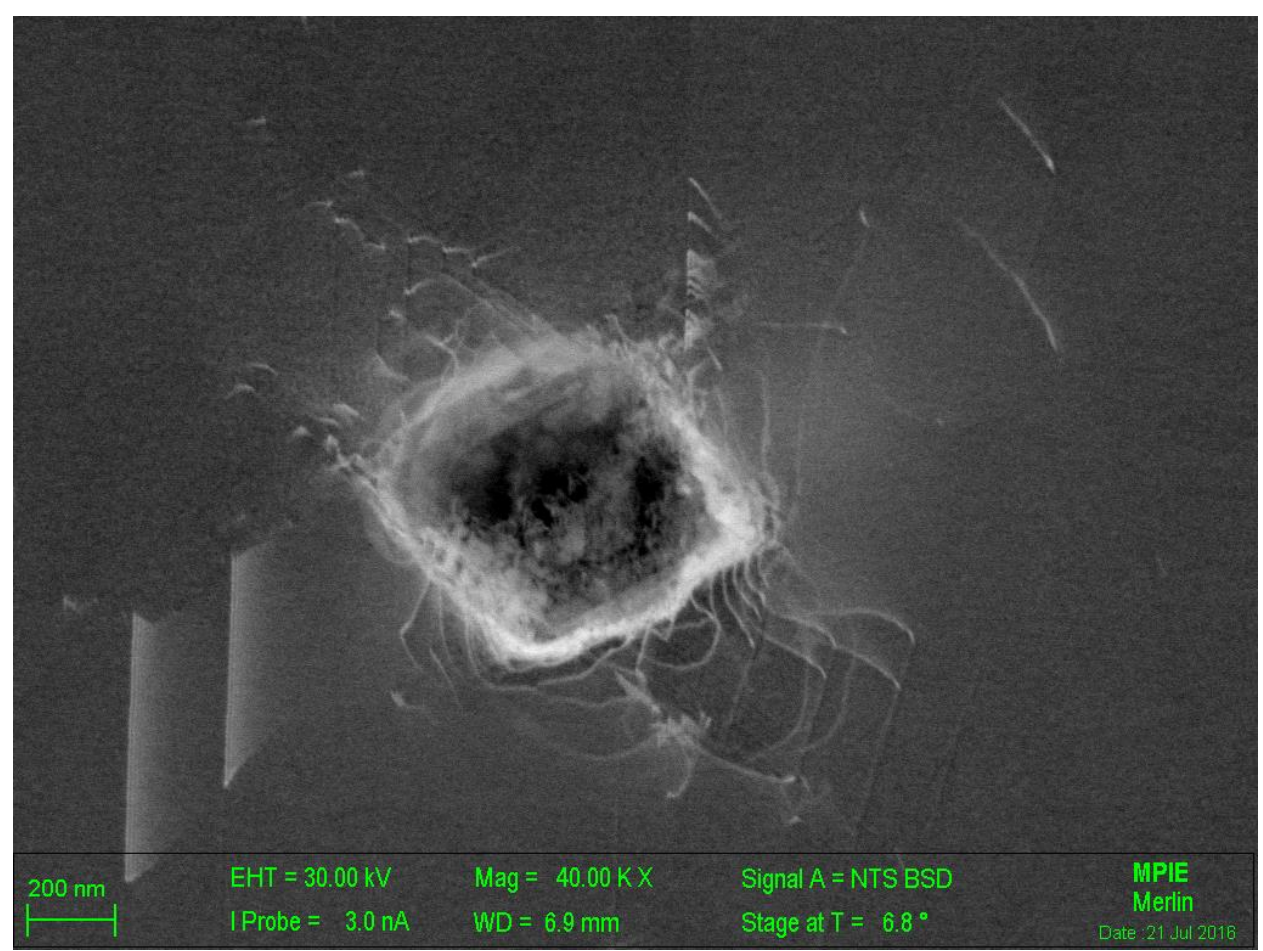

Figure 2: Dislocations and stacking faults formed around a nano-indent into a high-Mn steel sample

[1] S. Zaefferer, N.-N. Elhami, (2014) Theory and application of electron channelling contrast imaging under controlled diffraction conditions, Acta Materialia 75, 20-50.

[2] F. Ram, Z. Li, S. Zaefferer, M. Hafez Haghighat, Z. Zhu, D. Raabe, R. C. Reed.(2016), On the origin of creep dislocations in a Ni-base, single-crystal superalloy: an ECCI, EBSD, and Dislocation Dynamics-based study, Acta Materialia 109, 151-161 